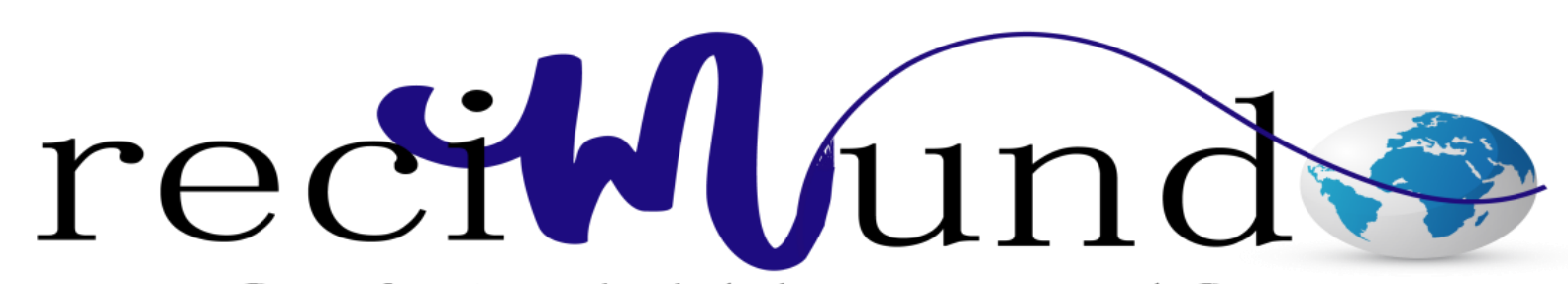

Revista Científica Mundo de la Investigación y el Conocimiento

Helen Irina Díaz Cedeño a; Maria Fernanda Monroy Pesantez ${ }^{\text {b; }}$ Darío Israel Macías

Ponce $^{\text {c; }}$ Ivan Leonardo Tuarez Villegas ${ }^{\mathrm{d}}$

Utilidad de la capnografía en urgencias

Usefulness of emergency capnography

Revista Científica Mundo de la Investigación y el Conocimiento. Vol. 3 núm.3. Esp., noviembre, ISSN: 2588-073X, 2019, pp. 218-238

DOI: $10.26820 /$ recimundo/3.(3.Esp).noviembre.2019.218-238

URL: http://recimundo.com/index.php/es/article/view/598

Código UNESCO: 3205 Medicina Interna

Tipo de Investigación: Artículo de Revisión

(C) RECIMUNDO; Editorial Saberes del Conocimiento, 2019

Recibido: 15/09/2019

Aceptado: 23/10/2019

Publicado: 30/11/2019

Correspondencia: helen_irina@hotmail.com

a. Médica; Investigadora Independiente; Guayaquil, Ecuador; helen_irina@hotmail.com

b. Médico; Investigador Independiente; Guayaquil, Ecuador; mafer.monroy309@ gmail.com

c. Médico; Investigador Independiente; Guayaquil, Ecuador; dario.macias.ponce@ gmail.com

d. Médico; Investigador Independiente; Guayaquil, Ecuador; ivletuvi1987@ gmail.com 
Utilidad de la capnografía en urgencias

Vol. 3, núm. 3 Esp., (2019)

Helen Irina Díaz Cedeño; Maria Fernanda Monroy Pesantez; Darío Israel Macías Ponce; Ivan

Leonardo Tuarez Villegas

\section{RESUMEN}

La capnografía, fue un descubrimiento que en 1895 hizo Joseph Black, químico que reveló las propiedades de un gas al que llamó "aire fijo" (refiriéndose al dióxido de carbono) que durante la espiración era soltado. Más recientemente se concibe como el método o proceso no invasivo y selectivo, que consiste en la monitorización complementaria a la pulsioximetría que facilita la evaluación de la ventilación de una persona, y que al mismo tiempo es dependiente de otros dos procesos de metabolización y perfusión. El objetivo de la presente investigación responde al interés por efectuar una revisión a la literatura científico-académica disponible libremente, mediante el uso de diversas bases de datos, a fines de constatar lo que los autores entendidos en la materia refieren sobre la utilidad que la capnografía representa en servicios de urgencias o emergencias. Los resultados comprenden la exposición sobre las concepciones fundamentales de la capnografía, fases del capnograma, métodos de medición, fisiopatología respiratoria, eventualidades clínicas detectables mediante la capnografía y utilidades e indicaciones en servicios de urgencias. En definitiva, se logró concluir que la capnografía representa un valiosísimo complemento de monitorización, que desde hace más de una década ha sido ponderada a nivel mundial un estándar de calidad al concebirse como una invaluable herramienta de apoyo para el correspondiente diagnóstico y valoración de los tratamientos administrados a pacientes con distintas patologías, tanto en los servicios de urgencia (emergencia) médica hospitalarios y extrahospitalarios, como también en otras unidades asistenciales, ya sean de cuidados críticos, reanimación, e inclusive, en unidades (ambulancias) móviles de emergencia.

Palabras Claves: Pulsioximetría; Monitorización; Metabolización; Perfusión; Función respiratoria. 


\title{
Utilidad de la capnografía en urgencias
}

Vol. 3, núm. 3 Esp., (2019)

Helen Irina Díaz Cedeño; Maria Fernanda Monroy Pesantez; Darío Israel Macías Ponce; Ivan Leonardo Tuarez Villegas

\begin{abstract}
The capnography was a discovery made in 1895 by Joseph Black, a chemist who revealed the properties of a gas called "fixed air" (referring to carbon dioxide) that was released during expiration. More recently it is conceived as the non-invasive and selective method or process, which consists of the complementary monitoring of pulse oximetry that facilitates the evaluation of a person's ventilation, and which at the same time is dependent on two other metabolization and perfusion processes. The objective of the present investigation responds to the interest in omitting a review of freely available scientific-academic literature, through the use of various databases, fines to verify what the authors understood in the matter related to the utility that the capnography represents in services of emergencies or emergencies. The results of the presentation on the fundamental conceptions of capnography, phases of the capnogram, measurement methods, respiratory pathophysiology, clinical eventualities detectable by capnography and utilities and indications in emergency departments. In conclusion, it is concluded that capnography represents a valuable monitoring complement, which for over a decade has been weighted a quality standard worldwide, conceived as an invaluable support tool for the corresponding diagnosis and evaluation of the treatments administered to patients with different pathologies, both in the emergency (emergency) hospital and out-of-hospital medical services, as well as in other care units, whether critical care, resuscitation, and even in mobile emergency (ambulance) units.
\end{abstract}

Keywords: Pulse oximetry; Monitoring; Metabolization; Perfusión; Respiratory function. 
Utilidad de la capnografía en urgencias

Vol. 3, núm. 3 Esp., (2019)

Helen Irina Díaz Cedeño; Maria Fernanda Monroy Pesantez; Darío Israel Macías Ponce; Ivan

Leonardo Tuarez Villegas

\section{Introducción.}

"La monitorización continua y no invasiva de la presión parcial de dióxido de carbono exhalado por el paciente a lo largo del tiempo" es lo que, según Pérez de Mendiguren (2017) refiere debe entenderse por capnografía.

En detalle, esta misma aclara que, es el proceso que facilita la evaluación de la ventilación de una persona, y que al mismo tiempo es dependiente de otros dos que ocurren con anticipación los cuales vienen siendo, primero, aquel en el que sucede la producción de $\mathrm{CO}$, llamado metabolismo, y luego, ocurriendo aquel en el que es trasladado el CO2 hasta los pulmones (conocido como perfusión), para así sucesivamente estos expulsen dicho gas al exterior del organismo. (p. 1)

Cereceda \& Molina (2017) en base a sus fuentes destacan que, como método electivo, la capnografía es concebida como coadyuvante para la evaluación del estado metabólico de los pacientes de manera no invasiva, inclusive, "en los cuidados de monitorización del paciente en diversas áreas asistenciales de anestesia y reanimación, cuidados críticos y emergencias" ha sido considerada un estándar de calidad desde haya ya un considerable tiempo. Así mismo han resaltado que, en momentos en los que en servicios de urgencias se requiere del diagnóstico y evaluación de los tratamientos administrados a pacientes con distintas patologías, es necesaria una gasometría arterial (GSA) o venosa (GSV) para evaluar el estado de oxigenación, ventilación y metabólico. En el mismo orden de ideas, afirman que es mediante la capnografía que se consigue "una monitorización complementaria a la pulsioximetría" en razón de que con esta "se dispone de información directa e inmediata de la ventilación, mientras que con la pulsioximetría 


\section{Utilidad de la capnografía en urgencias}

Vol. 3, núm. 3 Esp., (2019)

Helen Irina Díaz Cedeño; Maria Fernanda Monroy Pesantez; Darío Israel Macías Ponce; Ivan Leonardo Tuarez Villegas

se cuantifica únicamente la oxigenación" (p. 2).

A manera de reseña histórica sobre el uso de este tipo de evaluación clínica, Díez, Barrado, Blanco, Barroso, \& Espinosa (2009) describen que la misma se inició en pacientes intubados en medios hospitalarios de Europa, eso fue en el transcurso de los años 70, en base a los primeros estudios clínicos llevados a cabo por Smallhout y Kalenda, para en el siguiente decenio hacer lo propio con pacientes en los Estados Unidos de América. Luego, en el transcurrir de los años noventa y subsiguientes años del presente milenio, ésta medición fue cada vez más valorada y considerada por diversas instituciones de reconocido impacto en el área de la medicina y la salud, llegando al punto de que la Sociedad Americana de Anestesiólogos (ASA, por sus siglas en inglés) (1991) le considerara como "estándar de atención en el quirófano" a la par de la monitorización de la pulsioximetría; luego, en (1995) el Colegio Americano de Médicos de Emergencia (ACEP, por sus siglas en inglés) recomendó su uso de manera rutinaria en pacientes intubados tanto intra como extrahospitalariamente. La sugerencia de la Asociación Americana del Corazón (AHA, por sus siglas en inglés) (2000) fue la de que esta se usara "durante la parada cardiorrespiratoria y el tratamiento cardiovascular urgente" igualmente en pacientes atendidos tanto en el medio hospitalario como los del extrahospitalario. Es en 2002 que la Sociedad de Cuidados Intensivos (europea) determinó que este estudio sería un estándar de atención respecto al traslado, en el Reino Unido, de aquellos pacientes críticos de edad adulta. Para 2005, el Consejo Europeo de Reanimación (ERC, por sus siglas en inglés) confiaba en su uso para confirmar la colocación correcta del TET (tubo endotraqueal) al ocurrir una interrupción cardiorrespiratoria. Posteriormente, el Comité Europeo de Normalización en el año 2007 hizo su aporte en un aspecto relacionado, más no específicamente respecto a la capnografía, 


\section{Utilidad de la capnografía en urgencias}

Vol. 3, núm. 3 Esp., (2019)

Helen Irina Díaz Cedeño; Maria Fernanda Monroy Pesantez; Darío Israel Macías Ponce; Ivan

Leonardo Tuarez Villegas

cuando determino como estándar en Europa, la inclusión del capnómetro como parte del equipamiento necesario en ambulancias terrestres "tipo C", entendiéndose, aquellas "unidades móviles de emergencia o UVI móviles". (p. 139)

Esta obra responde al interés conjunto por efectuar una revisión la literatura científicoacadémica disponible libremente, mediante el uso de diversas bases de datos, a fines de constatar lo que los autores entendidos en la materia refieren sobre la utilidad que la capnografía representa en servicios de urgencias o emergencias, para ello, en lo sucesivo se ha dispuesto no solo conceptualizar la terminología básica relativa a la temática, sino también aquellos aspectos medico clínicos necesarios para la comprensión de los probables beneficios asociados a dicho proceso.

\section{Materiales y Métodos.}

El objetivo de esta investigación se delimitó a la búsqueda y revisión de literatura científico-académica reciente, disponible de manera gratuita mediante el uso de diversas bases de datos, entre las que figuran: NCBI, PubMed, MedlinePlus, SciELO, BVS, Embase, Dialnet, entre otras.

Básicamente, a exploración se hizo en base a las expresiones "Utilidad de la capnografía", "capnografía en urgencias" y "Utilidad de la capnografía en urgencias", tanto en español como en inglés, a fines de identificar, seleccionar y referir las fuentes que de la manera más resumida sirvan para explicar este tema.

El material bibliográfico que se consideró rescatar fue el relativo a: artículos científicos 


\section{Utilidad de la capnografía en urgencias}

Vol. 3, núm. 3 Esp., (2019)

Helen Irina Díaz Cedeño; Maria Fernanda Monroy Pesantez; Darío Israel Macías Ponce; Ivan Leonardo Tuarez Villegas

en general, guías clínicas, e-books, ensayos clínicos, consensos, protocolos, tesis de grado, posgrado y doctorado, noticias científicas, boletines y/o folletos de instituciones oficiales o privadas de reconocida trayectoria en el ámbito científico-académico y demás documentos e informaciones, considerados de interés y considerable valor de la evidencia científica a criterio propio, es decir, del equipo investigador, que se encontrasen, preferiblemente, publicados en los últimos 10 años (incluyendo el corriente), es decir, desde 2009 hasta el 2019, tomando en cuenta algunas excepciones.

Se desestimaron estudios de cohorte, casos y controles, series y reportes de casos, editoriales, cartas al editor y otros tipos de materiales bibliográficos que, a juicio de este equipo, se consideraron de escaso valor científico-académico o de bajo nivel de evidencia percibido en sus contenidos.

Tras la búsqueda, resultado obtenido fue superior a 500 referencias bibliográficas, en algunos casos, mientras que en otros ascendió a más de una decena de miles de fuentes documentales de diversa índole, eventos que en conjunto, demostró la suficiente existencia contenido desarrollado en base a esta temática, sin embargo, se manifestó igualmente evidente la necesidad de depurar dichos resultados, en función de los objetivos previamente planteados, por lo que progresivamente se aplicaron filtros en cuanto a: acceso completo y gratuito, tipo de literatura, idioma español o inglés, correlación y/o relevancia temática, publicación hecha dentro del lapso antes mencionado (exceptuando algunos), área de especialización o temática, entre otros.

Igualmente, se llevaron a cabo otras pesquisas sin mayores consideraciones a fines de 


\section{Utilidad de la capnografía en urgencias}

Vol. 3, núm. 3 Esp., (2019)

Helen Irina Díaz Cedeño; Maria Fernanda Monroy Pesantez; Darío Israel Macías Ponce; Ivan Leonardo Tuarez Villegas

encontrar aquella información igualmente relevante que, independientemente de su diferenciación para con el núcleo del tema, se considera necesaria para el desarrollo integral del mismo. Es entonces de allí en adelante que el equipo procedió con la lectura crítica y análisis interpretativo de toda la evidencia definitivamente escogida, resultando todo este proceso en la fundamentación del criterio aquí plasmado, por cierto, en todo momento hecho de manera consensuada.

Finalmente, es también destacable que, la exclusión e inclusión de cada una de las referencias hechas en la presente revisión se definió igualmente mediante el consenso grupal, y de la misma manera fueron resueltas las opiniones desiguales.

\section{Resultados.}

Pérez de Mendiguren (2017) con su trabajo aclara que la base de la capnografía es el descubrimiento que en 1895 hizo Joseph Black. Este químico reveló las propiedades de un gas al que llamó “aire fijo” (refiriéndose al dióxido de carbono) que durante la espiración era soltado. Posterior a este hecho es que en los años 70 surgen los primeros estudios por parte de Smallhout y Kalenda, comentados anteriormente, para así esta monitorización irse implementando sobre pacientes intubados en el medio hospitalario, primero en Europa, y en Estados Unidos, a partir de los años 80.

Desde la década de los 80, ha sido muy común la monitorización del CO2 exhalado mediante la capnografía en procedimientos que requerían de anestesia general, para detectar problemas en las vías respiratorias y prever tempranamente la disfunción pulmonar o cardíaca (p. $1-2)$ 


\section{Utilidad de la capnografía en urgencias}

Vol. 3, núm. 3 Esp., (2019)

Helen Irina Díaz Cedeño; Maria Fernanda Monroy Pesantez; Darío Israel Macías Ponce; Ivan Leonardo Tuarez Villegas

Por su parte, Llovet, Pichardo, \& Martínez (2016) en base a la idea de (Merino, 2015) legan que:

La Capnografía es un parámetro para la monitorización de la eficacia que está manteniendo una reanimación cardiopulmonar (RCP). Así, podemos valorar la si las compresiones torácicas están siendo efectivas al igual que la intubación endotraqueal, detectando de forma más rápida la circulación espontánea y ayudando a determinar el tiempo de las maniobras de resucitación empleadas.

Luego, los mismos tratadistas se fundamentan en la obra de (Morales, 2015) para indicar que:

El registro capnográfico o también llamado capnograma, es la representación de como ventila el paciente en un espacio de tiempo, en el eje vertical nos encontramos con las presiones parciales de dióxido de carbono $\left(\mathrm{CO}_{2}\right)$ medidos en milímetros de mercurio $(\mathrm{mmHg}) \mathrm{y}$ en el eje horizontal nos referimos al tiempo medidos en segundos.

Aunado a este contexto, se vuelve oportuno mencionar la acepción de Terradas (2015) quien aseguró que:

Una monitorización completa del ciclo respiratorio comprende la oximetría, valora la oxigenación y, la capnografía, nos aporta datos sobre la ventilación e incluso sobre el metabolismo y la perfusión, tanto en paciente intubado como no intubado.

Se considera oportuno mencionar la idea de Romero, Brunos, \& Arteaga (2019) quienes resaltaron que: 


\section{Utilidad de la capnografía en urgencias}

Vol. 3, núm. 3 Esp., (2019)

Helen Irina Díaz Cedeño; Maria Fernanda Monroy Pesantez; Darío Israel Macías Ponce; Ivan Leonardo Tuarez Villegas

[...] la evolución de la capnografía involucra al capnógrafo, el cual en la actualidad es común encontrar asociado a otros equipos que colaboran a la monitorización del paciente bajo anestesia o paciente crítico. Así mismo, se han desarrollado y software que permiten sintetizar toda la información que se registra por medio de estos aparatos e interactuar con esta para la toma de decisiones y la seguridad paciente. Lo más actualizado en esta materia incluye estudios de monitores inalámbricos, mucho más cómodos, no obstante, su valoración y requerimientos de internet los hacen inaccesibles para muchos países del mundo. (p. 90-91)

De esto pues, ahora es válido diferenciar que

\section{Fases del Capnograma (Fig.1)}

- Fase I: corresponde al período comprendido entre el final de la inspiración y el inicio de la espiración siguiente, cuando comienza la ventilación del espacio muerto formado por la vía aérea superior y parte del árbol bronquial que no tienen capacidad para intercambiar gases, y cuyo volumen de aire está prácticamente libre de $\mathrm{CO}_{2}$, siendo muy similar al del aire atmosférico. Al conectar el capnógrafo, éste reconoce esta presión de $\mathrm{CO}_{2}$ ambiental y la asimila al valor "cero", proceso conocido como "autocero", creando una línea isoeléctrica en el gráfico. (Fig. 1, segmento de curva A-B).

- Fase II: se inicia una rápida elevación gracias a la eliminación del $\mathrm{CO}_{2}$ del resto de espacio muerto, pero esta vez mezclado con el $\mathrm{CO}_{2}$ alveolar (Fig. 1, segmento de curva B-C).

- Fase III o meseta alveolar: el aire exhalado procede enteramente de los alvéolos, y 


\section{Utilidad de la capnografía en urgencias}

Vol. 3, núm. 3 Esp., (2019)

Helen Irina Díaz Cedeño; Maria Fernanda Monroy Pesantez; Darío Israel Macías Ponce; Ivan Leonardo Tuarez Villegas

se observa un ascenso lento y progresivo del $\mathrm{CO}_{2}$ que forma una meseta (Fig. 1, segmento de curva C-D), hasta alcanzar el punto en el que la presión parcial del gas es máxima (Fig. 1, punto D de la curva): éste es el valor presiométrico que registra el capnógrafo/capnómetro, el llamado $\mathrm{CO}_{2}$ teleespiratorio o $\mathrm{EtCO}_{2}$.

- Fase IV: comienza la fase inspiratoria y, por tanto, la presión parcial de CO2 decrece bruscamente hasta quedarse a cero (Fig. 1, segmento de curva D-E). (Barrado, Barroso, Patón, \& Sánchez, 2013) 


\section{Utilidad de la capnografía en urgencias}

Vol. 3, núm. 3 Esp., (2019)

Helen Irina Díaz Cedeño; Maria Fernanda Monroy Pesantez; Darío Israel Macías Ponce; Ivan Leonardo Tuarez Villegas

Figura 1. Capnograma Normal

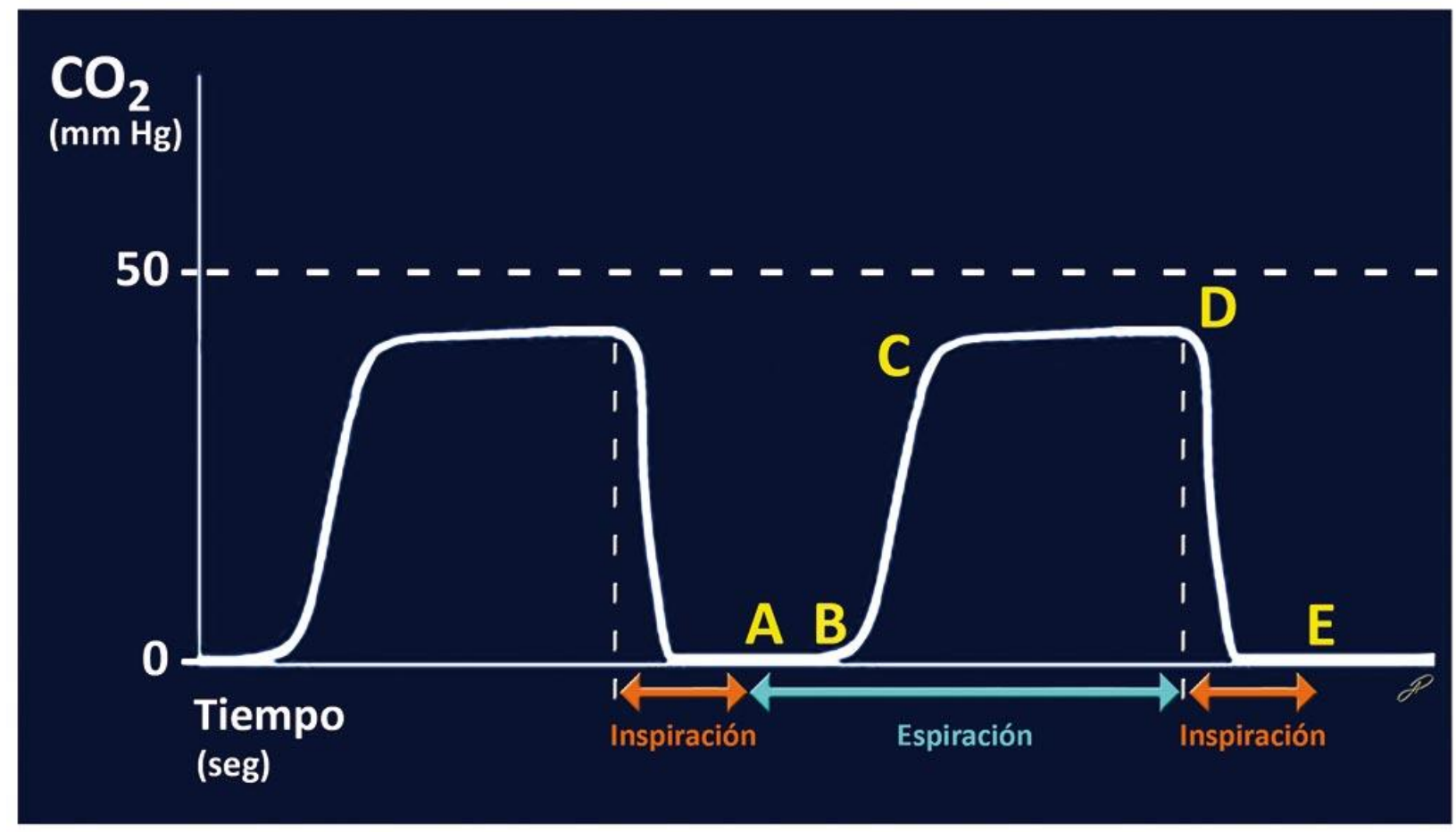

Nota. Recuperado de Barrado, Barroso, Patón, \& Sánchez (2013).

\section{Métodos de medición}

Sasal, Modrego, Morlán, \& Santafé (2017) hacen una necesaria diferenciación para no caer en confusión entre capnometría y capnografía. Aclaran que la capnometría está referida a la "medición del nivel de $\mathrm{CO}_{2}$ exhalado" siendo necesario para ello la utilización de un capnómetro; que por cierto muestra un valor numérico, solamente. En cambio, la capnografía, además mostrar el valor numérico del $\mathrm{CO}_{2}$ exhalado, "ofrece el registro gráfico de la eliminación de dicho $\mathrm{CO}_{2}$ a tiempo real y la frecuencia respiratoria". Es entonces que, el capnógrafo muestra de forma continua el $\mathrm{CO}_{2}$ exhalado (capnometría), el registro gráfico de la eliminación del mismo (capnograma) y la frecuencia respiratoria del paciente. 


\section{Utilidad de la capnografía en urgencias}

Vol. 3, núm. 3 Esp., (2019)

Helen Irina Díaz Cedeño; Maria Fernanda Monroy Pesantez; Darío Israel Macías Ponce; Ivan Leonardo Tuarez Villegas

La presión de $\mathrm{CO}_{2}$ espirada se determina por la absorción de luz infrarroja (espectrofotometría) o espectrometría de masas. En ella, los fragmentos iónicos del CO2 son atraídos por un campo magnético hacia los detectores; El muestreo puede determinarse de dos maneras:

- Muestreo central, en línea o main stream: con el detector directamente a nivel de salida del tubo. La lectura es sincrónica al movimiento del flujo de gases espirados.

- Muestreo lateral o side stream: mediante la aspiración del gas a nivel del tubo endotraqueal y con el detector a nivel del monitor. Existe un ligero retraso de $3-4$ segundos derivado de la demora de la llegada del gas al monitor.

Para la monitorización capnográfica, se requiere el análisis de gas espirado no contaminado, por lo que ha de evitarse la presencia de vapor de agua para evitar interferencias en la lectura. Aunque inicialmente se utilizaba exclusivamente en pacientes intubados, actualmente es posible realizar esta medición en pacientes no intubados; estos métodos de captación permiten simultáneamente administrar oxígeno sin que esto afecte a la medición de la capnografía. La evolución de la tecnología ha llevado a la medición por láser, ya que precisa menor aspiración de flujo para obtener resultados fiables, aunque actualmente se usa habitualmente en neonatos.

\section{Fisiopatología respiratoria}

Se hace necesario aclarar que, reiterando la exposición de Terradas (2015), lo que en síntesis comprende el ciclo respiratorio, para así entender mejor los procesos fisiológicos y fisiopatológicos asociados a la capnografía, y estos son: oxigenación, respiración celular aeróbica (ciclo de Krebs) y ventilación. 


\section{Utilidad de la capnografía en urgencias}

Vol. 3, núm. 3 Esp., (2019)

Helen Irina Díaz Cedeño; Maria Fernanda Monroy Pesantez; Darío Israel Macías Ponce; Ivan Leonardo Tuarez Villegas

El experto explica que el proceso de oxigenación inicia con el ingreso de aire a los pulmones mediante la vía aérea, una vez allí, en razón de la diferencia de gradiente de presión, el oxígeno $\left(\mathrm{O}_{2}\right)$ se difunde a través de las paredes de los alveolos hacia la sangre de los capilares que lo recubren, luego el $\mathrm{O}_{2}$ se une de forma reversible a la hemoglobina $(\mathrm{Hb})$ convirtiéndose así en oxihemoglobina $\left(\mathrm{HbO}_{2}\right)$ que seguidamente será transportada por el torrente sanguíneo a las células de organismo, siendo este proceso en resumen monitorizado con la oximetría.

En lo sucesivo se da el ciclo de Krebs (respiración celular aeróbica), tratándose de que el $\mathrm{O}_{2}$ transportado por la $\mathrm{Hb}$ difunde al interior de la mitocondria el, así $\mathrm{O}_{2}$ actúa en la degradación de las biomoléculas (glucosa, lípidos y proteínas) liberando energía al generar moléculas de adenosín trifosfato (ATP) necesarias para el funcionamiento de las células y productos de deshecho $\left(\mathrm{H}_{2} \mathrm{O}\right.$ y $\left.\mathrm{CO}_{2}\right)$, entonces, una porción del $\mathrm{CO}_{2}$ se convierte en ácido carbónico $\left(\mathrm{H}_{2} \mathrm{CO}_{3}\right)$ que a la vez se ioniza formando bicarbonato (en un 70\%) y otra parte es transportado por la $\mathrm{Hb}$ en dirección a los pulmones en forma de carbohemoglobina $\left(\mathrm{HbCO}_{2}\right)$.

En último lugar ocurre la ventilación, en la que la carbohemoglobina $\left(\mathrm{HbCO}_{2}\right)$ contenida en los pulmones, mediante los alveolos, difundirá el CO2 al aire exhalado por las vías aéreas, siendo posible su monitorización precisamente mediante capnografía.

Este experto, respecto a la relación existente el ciclo antes descrito y la capnografía, adicionalmente declara que esta, además de monitorizar la eliminación de $\mathrm{CO}_{2}$ en los pulmones (la ventilación), servirá para valorar el transporte de $\mathrm{CO}_{2}$ por el torrente sanguíneo hasta los pulmones (la perfusión) y/o la producción de $\mathrm{CO}_{2}$ celular (el metabolismo) del paciente cuando 


\section{Utilidad de la capnografía en urgencias}

Vol. 3, núm. 3 Esp., (2019)

Helen Irina Díaz Cedeño; Maria Fernanda Monroy Pesantez; Darío Israel Macías Ponce; Ivan Leonardo Tuarez Villegas

dos de estas tres mediciones se mantengan estables o una interpretación conjunta en caso contrario.

Eventualidades clínicas detectables con la capnografía

Del aporte de Morales (2014) y Hormeño (2018) se extrae que, mediante la capnografía pueden ser detectadas anticipadamente los siguientes eventos clínicos:

- Hipoventilación

- Intubación bronquial.

- $\quad$ Re-Respiración parcial.

- Broncoespasmo.

- $\quad$ Fuga en el circuito.

- $\quad$ Paro cardiaco o PCR.

- Extubación accidental.

- Embolismo pulmonar.

- Desconexión del circuito.

- $\quad$ Hipertermia maligna.

- Intubación esofágica.

- $\quad$ Laringoespasmo.

- $\quad$ Obstrucción aérea parcial.

- $\quad$ TEP e IOT.

En el mismo orden de ideas, de Sasal et al. (2017) también se logra destacar el hecho de 


\section{Utilidad de la capnografía en urgencias}

Vol. 3, núm. 3 Esp., (2019)

Helen Irina Díaz Cedeño; Maria Fernanda Monroy Pesantez; Darío Israel Macías Ponce; Ivan

Leonardo Tuarez Villegas

que, si bien es cierto que la capnografía es usada complementariamente a la pulsioximetría para el completo monitoreo de la función respiratoria del paciente, es igualmente verídico el retardo de 2 a 4 minutos en la detección de eventos graves cuando solamente se procede solamente con la pulsioximetría, de pues bien se pudiera evidenciar la importancia de la capnografía, en virtud de que:

[...] ofrece información sobre la concentración de $\mathrm{CO}_{2}$ al final de la espiración, frecuencia y ritmo respiratorio, cálculo del espacio muerto, gasto cardiaco, confirmación de la colocación y posible obstrucción del tubo endotraqueal y la presencia de enfermedad obstructiva de las vías aéreas.

\section{Utilidades e Indicaciones}

En la obra de Morales (2014) se explica que los capnógrafos están compuestos por dispositivos que permiten vigilar a cualquier clase de paciente, desde adultos hasta neonatos, ya sea que se encuentren con ventilación asistida (intubados) o con respiración espontánea (ni intubados), y aunque estos representen un modo de monitorización no invasiva, no significa que con los mismos no se pueda sacar un mayor provecho en múltiples aplicaciones clínicas tal y como otros equipos de monitorización invasivos, en efecto las utilidades destacables de la capnografía en urgencias podría ser:

- Confirmación y control de la adecuada posición del tubo endotraqueal (TET); porque su correcta colocación es verificable al observar a lo largo del tiempo el mantenimiento de los niveles capnométricos y un capnograma normal, y en caso contrario, el escaso $\mathrm{CO} 2$ residual, incluso a veces inexistente, en el tracto digestivo 


\section{Utilidad de la capnografía en urgencias}

Vol. 3, núm. 3 Esp., (2019)

Helen Irina Díaz Cedeño; Maria Fernanda Monroy Pesantez; Darío Israel Macías Ponce; Ivan Leonardo Tuarez Villegas

alto provocaría la aparición de valores capnométricos y curvas capnográficas muy bajos y decrecientes hasta llegar a cero en un intervalo muy corto de tiempo. También es evidenciable por los frecuentes errores cometidos con la intubación selectiva, y que trata de la exagerada introducción del TET (por lo general en el bronquio principal derecho), con lo que se deja sin ventilar el pulmón contralateral.

- Control de la terapia respiratoria; debido a que en pacientes con ventilación mecánica invasiva (VMI) o no invasiva (VMNI), pueden verse comprometidos por los múltiples efectos adversos que se pueden presentar a nivel respiratorio, cardiovascular, neurológico, renal, digestivo, u otros, imputables en gran medida a la presión positiva intratorácica que ejerce el soporte ventilatorio mecánico.

- Control, progreso y pronóstico de la reanimación cardiopulmonar; que en síntesis se traduce en la verificación de la correcta colocación del TET, valoración de la calidad del masaje cardíaco, indicador temprano de la recuperación de la circulación espontánea (ROSC, por sus siglas en inglés) y pronóstico de la reanimación.

- Monitorización diagnóstica y terapéutica del asma y la enfermedad pulmonar obstructiva crónica (EPOC) reagudizada; ya que se puede valorar en tiempo real (gracias al estudio de las tendencias capnométricas/capnográficas) los cambios ventilatorios que presenta el paciente durante la asistencia médica, es decir, confirmar la eficacia o no del tratamiento pautado.

- Monitorización de las alteraciones del patrón respiratorio; debido a que, cuando se usa de forma conexa con la oximetría de pulso, permite valorar de forma continua la 


\section{Utilidad de la capnografía en urgencias}

Vol. 3, núm. 3 Esp., (2019)

Helen Irina Díaz Cedeño; Maria Fernanda Monroy Pesantez; Darío Israel Macías Ponce; Ivan Leonardo Tuarez Villegas

función y el patrón respiratorio del paciente, aspecto sumamente útil para descubrir cuadros de, por ejemplo: hipoventilación, a su vez procedentes de procesos seudoanalgésicos mal controlados o a intoxicaciones por alcohol, drogas, fármacos, u otros, lo que por consiguiente ayuda al especialista en la elección del tratamiento médico a emplear.

- Monitorización complementaria en estados de baja perfusión; puesto que, al combinarlo con la monitorización de la presión arterial y la frecuencia cardíaca, se podrá evidenciar un descenso brusco de los valores registrados en casos de hipovolemias súbitas (rotura de aneurisma, rotura esplénica, entre otras) o tromboembolia pulmonar, eventos que se encuentras asociados con un estado hemodinámico.

- Estados metabólicos alterados; en razón de que permite valorar la respuesta al tratamiento de la hipotermia, tanto accidental, como terapéutica, detectar de forma temprana acidosis metabólicas en pacientes con gastroenteritis aguda, fundamentalmente en niños, así como cuadros de deshidratación y cetoacidosis diabética.

En definitiva, y en concordancia con el aporte de Cereceda \& Molina (2017), se cree que en la actualidad, al parecer, la evidencia científica continúa apoyando el uso cada vez mayor de la capnografía, como "nuevo signo, biomarcador o parámetro centinela complementario en la detección de aquellos pacientes que se encuentran en una situación de enfermedad grave y es fácilmente implementable en aquellos pacientes que están en respiración espontánea" (p. 8). Así mismo, estos especialistas concluyeron que cuando se obtienen valores capnográficos bajos en 


\section{Utilidad de la capnografía en urgencias}

Vol. 3, núm. 3 Esp., (2019)

Helen Irina Díaz Cedeño; Maria Fernanda Monroy Pesantez; Darío Israel Macías Ponce; Ivan Leonardo Tuarez Villegas

pacientes con síntomas y características asociadas a alguna patología emergente (sobre todo menores a $24.5 \mathrm{mmHg}$ ), es probable que sirvan de manera temprana para llevar a cabo nuevas pruebas que arrojen resultados más exactos para de esa manera superar posibles retrasos en la asistencia y minimizar las tasas de morbilidad y mortalidad en el área de urgencias (emergencias). (p.8-9)

\section{Conclusiones.}

Es indiscutible el reconocimiento de la utilidad que representa la capnografía en urgencias ya que, como se ha procurado dar a entender durante el desarrollo de esta revisión, por una parte, representa un valiosísimo complemento de monitorización de la pulsioximetría, y por la otra, no menos trascendente es el hecho de que, desde hace más de diez años a nivel mundial ha sido igualmente ponderada como una invaluable herramienta de apoyo para el correspondiente diagnóstico y valoración de los tratamientos administrados a pacientes con distintas patologías, tanto en los servicios de urgencia (emergencia) médica hospitalarios y extrahospitalarios, como también en otras unidades asistenciales, ya sean de cuidados críticos, reanimación e inclusive, gracias a los constantes avances tecnológicos, en unidades (ambulancias) móviles de emergencia, de allí pues sirva para comprender su concepción como estándar de calidad en estos servicios.

Entendiendo que clínicamente la capnografía puede ser propia de diversas indicaciones, resulta que en base a la literatura consultada y los estudios en ellas referidos indican que en general las más destacadas son: a) la monitorización de la RCP, $b$ ) la clasificación, la valoración y el control del tratamiento en las crisis de broncoespasmo, y (en urgencias especialmente, c) el 


\section{Utilidad de la capnografía en urgencias}

Vol. 3, núm. 3 Esp., (2019)

Helen Irina Díaz Cedeño; Maria Fernanda Monroy Pesantez; Darío Israel Macías Ponce; Ivan Leonardo Tuarez Villegas

control de la colocación correcta del TET y $d$ ) la detección oportuna de la extubación accidental durante un traslado.

\section{Bibliografía.}

Barrado, L., Barroso, S., Patón, G., \& Sánchez, J. (2013). Capnografía, la evolución en la monitorización del paciente crítico. Zona TES, 2(1), 1-7.

Cereceda, F., \& Molina, J. (2017). Capnografía como herramienta para detectar alteraciones metabólicas de los pacientes atendidos en situaciones de urgencia. Revista LatinoAmericana de Enfermedades, 25(2885), 1-10 .

Díez, L., Barrado, L., Blanco, P., Barroso, S., \& Espinosa, S. (2009). La capnografía en los servicios de emergencia médica. SEMERGEN, 35(3), 138-143.

Hormeño, R. (15 de Mayo de 2018). Aplicaciones de la capnografía en servicios de urgencias. Obtenido de Capnografía en urgencias: http://www.areasaludbadajoz.com/images/stories/capnografia_urgencias_2018.pdf

Llovet, L., Pichardo, I., \& Martínez, C. (2016). Capnografía como instrumento útil para los profesionales enfermeros en Atención Primaria en situaciones de emergencias. En Comps., M. Pérez Fuentes, M. Molero, J. Gázquez, A. Barragán, Á. Martos, M. Pérez Esteban, \& A. G. Salvador (Ed.), Cuidados, aspectos psicológicos y actividad físisca en relación con la salud (Vol. II, pág. 690 ). Asociación Universitaria de Educación y Psicología (ASUNIVEP).

Morales, M. (2014). Uso de la capnografía en urgencias. Universidad Internacional de Andalucía. Andalucia: Universidad Internacional de Andalucía. Recuperado el 05 de 10 de 2019, de https://dspace.unia.es/bitstream/handle/10334/3425/0610_Morales.pdf?sequence=3\&isAl lowed $=\mathrm{y}$

Pérez de Mendiguren, P. (2017). Guía rápida y póster sobre capnografía. Obtenido de Academica-e.U navarra: https://academicae.unavarra.es/bitstream/handle/2454/26688/TFG\%20Paula\%20P\%c3\%a9rez\%20de\%20 Mendiguren.pdf? sequence $=2 \&$ is Allowed $=\mathrm{y}$

Romero, M., Brunos, C., \& Arteaga, N. (Septiembre de 2019). La capnografía: estudio fundamental en el monitoreo del paciente anestesiado. RECIAMUC, 3(3), 82-95.

Sasal, S., Modrego, D., Morlán, S., \& Santafé, S. (21 de enero de 2017). Conociendo la capnografía. Revista Electrónica de PortalesMedicos, 7(2). 


\section{Utilidad de la capnografía en urgencias}

Vol. 3, núm. 3 Esp., (2019)

Helen Irina Díaz Cedeño; Maria Fernanda Monroy Pesantez; Darío Israel Macías Ponce; Ivan Leonardo Tuarez Villegas

Terradas, J. (31 de Octubre de 2015). Capnografia en adultos en urgencias y emergencias. Obtenido de SemesBaleares: http://www.semesbaleares.org/colectivo.aspx?colectivo=2\&idtiponoticia=1\&IDNOTICI $\mathrm{A}=47 \& \mathrm{PAG}=0 \& \mathrm{f}=\&$ IDPUBLICACION=44\#publicacion

$$
\text { (9) }(1)(9)
$$

RECONOCIMIENTO-NOCOMERCIAL-COMPARTIRIGUAL

CC BY-NC-SA

ESTA LICENCIA PERMITE A OTROS ENTREMEZCLAR, AJUSTAR Y CONSTRUIR A PARTIR DE SU OBRA CON FINES NO COMERCIALES, SIEMPRE Y CUANDO LE RECONOZCAN LA AUTORÍA Y SUS NUEVAS CREACIONES ESTÉN BAJO UNA LICENCIA CON LOS MISMOS TÉRMINOS. 